\title{
Critical points of asymptotically quadratic functions
}

\author{
by Michal FEČKAN (Bratislava)
}

\begin{abstract}
Existence results for critical points of asymptotically quadratic functions defined on Hilbert spaces are studied by using Morse-Conley index and pseudomonotone mappings. Applications to differential equations are given.
\end{abstract}

1. Introduction. In this paper, we study the existence of critical points for functions on infinite-dimensional spaces which are asymptotically quadratic at infinity. Recently, several papers dealing with such problems have appeared. The so-called P.S. condition is required in most of those papers. First results of that kind have been obtained by H. Amann and E. Zehnder (see $[1,4,5])$.

In Section 2, we investigate functions asymptotically quadratic at infinity with pseudomonotone gradients. The linear asymptotes of the gradients at infinity are allowed to be either invertible or not. Such functions can be approximated by functions satisfying the P.S. condition. So we are able to combine some results on the Morse-Conley index (see [1, 4]) and on pseudomonotone mappings (see $[6]$ ). We were stimulated by $[2,3]$ to use that approximation (non-Galerkin) method. By using that method, we also hint at a possible way of extending the theory of Morse-Conley index of [1] to functions asymptotically quadratic at infinity with pseudomonotone gradients. Results on the existence of nontrivial critical points are derived as well, i.e. we deal with the existence of additional critical points of functions with a finite number of critical points. Consequently, we give a generalization of $\left[4\right.$, Theorem $\left.1.2^{\prime}\right]$. Generally, when gradients of functions are only pseudomonotone, we are almost able to find nontrivial critical points. To find exact nontrivial critical points, we have to require the so-called property $S_{+}$(see [3, p. 946]) of gradients. The property $S_{+}$is stronger than pseudomonotony.

1991 Mathematics Subject Classification: 58E05, 58E99.

Key words and phrases: critical points, Morse-Conley index, pseudomonotone mappings. 
In Section 3, we use these results to show the existence of weak solutions for the following two differential equations:

$$
\begin{aligned}
& \frac{\partial F}{\partial x}\left(t, u(t), u^{\prime}(t)\right)-\frac{\partial}{\partial t}\left(\frac{\partial F}{\partial y}\left(t, u(t), u^{\prime}(t)\right)\right)=0, \\
& u: \mathbb{R} \rightarrow \mathbb{R} \text { is } 2 \pi \text {-periodic, }
\end{aligned}
$$

and

$$
\begin{aligned}
& -\sum_{i=1}^{n} \frac{\partial}{\partial x_{i}}\left(f_{i}(x, \nabla u)\right)+g(x, u)=0, \quad x \in \Omega \subset \mathbb{R}^{n}, \\
& u(x)=0, \quad x \in \partial \Omega, u: \Omega \rightarrow \mathbb{R},
\end{aligned}
$$

where $F \in C^{1}(\mathbb{R} \times \mathbb{R} \times \mathbb{R}, \mathbb{R})$ is $2 \pi$-periodic in $t, f_{i}(x, y)=\frac{\partial \bar{F}}{\partial y_{i}}(x, y), \bar{F} \in$ $C^{2}\left(\mathbb{R}^{n} \times \mathbb{R}^{n}, \mathbb{R}\right), g \in C^{0}\left(\mathbb{R}^{n} \times \mathbb{R}, \mathbb{R}\right)$ and $\Omega$ is an open bounded subset of $\mathbb{R}^{n}$ with a smooth boundary $\partial \Omega$.

2. Abstract results. Let $H$ be a Hilbert space with an inner product $\langle\cdot, \cdot\rangle$ and the norm $|\cdot|$. Note that the index of a bounded self-adjoint linear map is the (finite) dimension of the subspace of all eigenvectors of that map with negative eigenvalues. The index of a critical point of a $C^{2}$-smooth function is the index of its Hessian at that point. The Hessian at a critical point $x$ of a $C^{2}$-smooth function $f$ is denoted by Hess $f(x)$. A critical point $x$ of a $C^{1}$-smooth function $f$ is nondegenerate if $f$ is $C^{2}$-smooth at $x$ and Hess $f(x)$ is invertible.

THEOREM 2.1. Let $f \in C^{1}(H, \mathbb{R})$ and suppose that

(i) $f(u)=\frac{1}{2}\langle L u, u\rangle+g(u)$, where $L$ is an invertible self-adjoint bounded linear operator for which the index exists;

(ii) $\operatorname{grad} g(x)=o(|x|)$ as $|x| \rightarrow \infty$;

(iii) $\operatorname{grad} f$ is pseudomonotone (see [3, p. 946]).

Then there is a solution of $\operatorname{grad} f(u)=0$.

Proof. Let us consider $f_{\varepsilon}(u)=f(u)+\varepsilon|u|^{2} / 2$ for $\varepsilon>0$ small. So $\operatorname{grad} f_{\varepsilon}=\operatorname{grad} f+\varepsilon \mathbb{I}$. Since it is the sum of a pseudomonotone mapping with $\varepsilon \mathbb{I}$ for $\varepsilon>0$, we know by $[2,3,6]$ that $\operatorname{grad} f_{\varepsilon}$ satisfies (see also Definition 2.4 below)

$$
\text { if } u_{n} \rightarrow u \text { and } \varlimsup_{n \rightarrow \infty}\left\langle\operatorname{grad} f_{\varepsilon}\left(u_{n}\right)-\operatorname{grad} f_{\varepsilon}(u), u_{n}-u\right\rangle \leq 0 \text {, then } u_{n} \rightarrow u
$$

(see [6, Definition 3.3.16]). Here $\rightarrow$ means weak convergence.

Furthermore, $\operatorname{grad} f_{\varepsilon}(x)=(L+\varepsilon \mathbb{I}) x+o(|x|)$ as $|x| \rightarrow \infty$. These two properties of $\operatorname{grad} f_{\varepsilon}$ imply the validity of the P.S. condition for any $\varepsilon>0$ sufficiently small. Indeed, it is sufficient to observe the following fact: the P.S. condition is satisfied for a function $\widetilde{f} \in C^{1}(H, \mathbb{R})$ provided that grad $\widetilde{f}$ has the above property (see Definition 2.4 below) and $(\operatorname{grad} \widetilde{f})^{-1}(B)$ is bounded 
for any bounded subset $B$. The last property is valid if $\operatorname{grad} \tilde{f}$ has a linear invertible asymptote at infinity.

Since $\left\|(L+\varepsilon \mathbb{I})^{-1}\right\|$ is uniformly bounded for $\varepsilon$ small, by applying the well-known result of $[1,4]$, there is a constant $K>0$ and $u_{\varepsilon} \in H$ such that $\operatorname{grad} f_{\varepsilon}\left(u_{\varepsilon}\right)=0$ and $\left|u_{\varepsilon}\right| \leq K$ for any $\varepsilon>0$ small. So grad $f\left(u_{\varepsilon}\right)=-\varepsilon u_{\varepsilon} \rightarrow 0$ as $\varepsilon \rightarrow 0_{+}$. By using the pseudomonotony of $\operatorname{grad} f$, the existence of a solution of $\operatorname{grad} f(u)=0$ is standardly proved (see [6]).

Definition 2.2. An equation $F(u)=0, F: S \rightarrow H$, is said to be almost solvable on $S \subset H$ if $0 \in \overline{F(S)}$, i.e. there is a sequence $\left\{u_{n}\right\}_{n=1}^{\infty} \subset S$ such that $F\left(u_{n}\right) \rightarrow 0$ as $n \rightarrow \infty$.

Theorem 2.3. Suppose $f$ satisfies the conditions (i)-(iii) of Theorem 2.1. Moreover, assume that

(i) $\operatorname{grad} f(0)=0$ and $A=\operatorname{Hess} f(0)$ exists, it is invertible and index $A$ exists;

(ii) index $A \neq \operatorname{index} L$.

Then there are open, bounded neighbourhoods $U_{1}, U_{2}$ of 0 such that $U_{1} \subset U_{2}$ and $\operatorname{grad} f=0$ is almost solvable on $U_{2} \backslash U_{1}$. (We then say that $\operatorname{grad} f=0$ almost has a nonzero solution.)

Proof. We follow the above proof. Since index $(L+\varepsilon \mathbb{I}) \neq \operatorname{index}(A+\varepsilon \mathbb{I})$ for $\varepsilon$ small, by using again the well-known result of $[1,4]$, there are positive constants $K, k$ and $u_{\varepsilon} \in H$ such that $\operatorname{grad} f_{\varepsilon}\left(u_{\varepsilon}\right)=0$ and $k \leq\left|u_{\varepsilon}\right| \leq K$ for any $\varepsilon>0$ small. So grad $f\left(u_{\varepsilon}\right)=-\varepsilon u_{\varepsilon} \rightarrow 0$ as $\varepsilon \rightarrow 0_{+}$. The proof is finished in the same way as for Theorem 2.1.

Definition 2.4. An operator $F: H \rightarrow H$ has the property $S_{+}$if whenever $u_{n} \rightarrow u$ and

$$
\varlimsup_{n \rightarrow \infty}\left\langle\operatorname{grad} f\left(u_{n}\right)-\operatorname{grad} f(u), u_{n}-u\right\rangle \leq 0,
$$

then $u_{n} \rightarrow u$ (see [6, Definition 3.3.16], [3, p. 946]).

We already know that $f \in C^{1}(H, \mathbb{R})$ satisfies the P.S. condition provided that $\operatorname{grad} f$ has the property $S_{+}$and $(\operatorname{grad} f)^{-1}(B)$ is bounded for any bounded subset $B$. The last property is valid if grad $f$ has a linear invertible asymptote at infinity. This implies that if $\operatorname{grad} f$ has the property $S_{+}$in Theorem 2.3, which is stronger than pseudomonotony, then $\operatorname{grad} f=0$ has a nonzero solution. This result is well known (see $[1,4])$.

If $f \in C^{1}(H, \mathbb{R})$ is such that $f(u)=\frac{1}{2}\langle L u, u\rangle+g(u)$ for a bounded linear self-adjoint operator $L$ with $\operatorname{ker} L \neq\{0\}$, the assumption (ii) of Theorem 2.1 is satisfied as well, and grad $f$ has the property $S_{+}$, then $f$ generally does not satisfy the P.S. condition.

Now we extend Theorems 2.1 and 2.3 to resonant cases. 
THEOREM 2.5. Let $L: H \rightarrow H$ be a Fredholm, self-adjoint bounded linear operator for which the index exists and $\operatorname{dim} \operatorname{ker} L>0$. Let $H_{1} \oplus \operatorname{ker} L=H$ be the orthogonal decomposition with the orthogonal projection $P: H \rightarrow \operatorname{ker} L$. Suppose that $f \in C^{1}(H, \mathbb{R})$ satisfies the following assumptions:

(i) $f(u)=\frac{1}{2}\langle L u, u\rangle+g(u)$;

(ii) there is a constant $M>0$ such that $|\operatorname{grad} g(x)| \leq M$ for all $x \in H$;

(iii) $\operatorname{grad} f$ is pseudomonotone;

(iv) there is a continuous map $\omega: S_{1}=\{v \in \operatorname{ker} L|| v \mid=1\} \rightarrow H$ such that

$$
\lim _{r \rightarrow+\infty} \operatorname{grad} g(u+r v)=\omega(v)
$$

uniformly in both $v \in S_{1}$ and $u \in \mathcal{A}$ for any fixed bounded subset $\mathcal{A}$ of $H_{1}$;

(v) there is no solution of the equation $\lambda v+P \omega(v)=0$ with $v \in S_{1}$ and $\lambda \geq 0$.

Then there is a solution of $\operatorname{grad} f(u)=0$.

Proof. Let us consider $f_{\varepsilon}(u)=f(u)+\varepsilon|u|^{2} / 2$ for $\varepsilon>0$ small. We already know by the proof of Theorem 2.1 that there is $u_{\varepsilon} \in H$ such that $\operatorname{grad} f_{\varepsilon}\left(u_{\varepsilon}\right)=0$ for any $\varepsilon>0$ small. Now we show the boundedness of $\left\{u_{\varepsilon}\right\}$ by using some ideas of [7]. Assume $u_{\varepsilon} \rightarrow \infty$. We take the orthogonal projections $P: H \rightarrow \operatorname{ker} L$ and $Q=\mathbb{I}-P$. Then

$$
\begin{aligned}
& (L+\varepsilon \mathbb{I}) u_{\varepsilon 2}+Q \operatorname{grad} g\left(u_{\varepsilon}\right)=0, \\
& \varepsilon u_{\varepsilon 1}+P \operatorname{grad} g\left(u_{\varepsilon 1}+u_{\varepsilon 2}\right)=0, \\
u_{\varepsilon}= & u_{\varepsilon 1}+u_{\varepsilon 2}, \quad u_{\varepsilon 1} \in \operatorname{ker} L, \quad u_{\varepsilon 2} \in H_{1} .
\end{aligned}
$$

The assumption (ii) implies the boundedness of $\left\{u_{\varepsilon 2}\right\}$ and $\left\{\varepsilon u_{\varepsilon 1}\right\}$. So $u_{\varepsilon 1} \rightarrow$ $\infty$. By putting $w_{\varepsilon}=u_{\varepsilon 1} /\left|u_{\varepsilon 1}\right|$ we have

$$
\varepsilon\left|u_{\varepsilon 1}\right| w_{\varepsilon}+P \operatorname{grad} g\left(u_{\varepsilon 2}+\left|u_{\varepsilon 1}\right| w_{\varepsilon}\right)=0 .
$$

We can assume $w_{\varepsilon} \rightarrow w_{0}$ and $\varepsilon\left|u_{\varepsilon 1}\right| \rightarrow \lambda_{0}$. Finally, we arrive at the equation

$$
\lambda_{0} w_{0}+P \omega\left(w_{0}\right)=0
$$

for some $w_{0} \in S_{1}$ and $\lambda_{0} \geq 0$. The contradiction with the assumption (v) proves the boundedness of $\left\{u_{\varepsilon}\right\}$. The rest of the proof is similar to that for Theorem 2.1.

THEOREM 2.6. Suppose $f$ satisfies the conditions (i)-(ii), (iv)-(v) of Theorem 2.5. Moreover, assume

(i) grad $f$ has the property $S_{+}$;

(ii) $\operatorname{grad} f(0)=0$ and $A=\operatorname{Hess} f(0)$ exists, it is invertible and index $A$ exists;

(iii) index $A \neq \operatorname{index} L$

Then $\operatorname{grad} f=0$ has a nonzero solution. 
Proof. We follow the proofs of Theorems 2.3 and 2.5. So there are positive constants $K, k$ and a sequence $\left\{u_{\varepsilon}\right\}$ such that $\operatorname{grad} f_{\varepsilon}\left(u_{\varepsilon}\right)=0$ and $k<\left|u_{\varepsilon}\right|<K$ for any $\varepsilon>0$ small. Since grad $f$ has the property $S_{+}$, we can assume $u_{\varepsilon} \rightarrow u_{0}$. The proof is finished.

Remark 2.7. The variational nature of the problems in Theorems 2.1 and 2.5 is not essential. By using ideas of $[2,3]$, it is possible to derive nonvariational versions of these theorems. The assumptions (iv)-(v) of Theorem 2.5 are the so-called Landesman-Lazer conditions (see [7]).

Remark 2.8. If $f \in C^{1}(H, \mathbb{R})$ satisfies $f(u)=\frac{1}{2}\langle L u, u\rangle+g(u)$, where $L$ is a Fredholm, self-adjoint bounded linear operator for which the index exists and $\operatorname{grad} g$ is compact, then $\operatorname{grad} f$ has the property $S_{+}$. Indeed, assume $u_{n} \rightarrow u$ and

$$
\varlimsup_{n \rightarrow \infty}\left\langle\operatorname{grad} f\left(u_{n}\right)-\operatorname{grad} f(u), u_{n}-u\right\rangle \leq 0 .
$$

Then $\left\{u_{n}\right\}_{n=1}^{\infty}$ is bounded. Hence we can assume the existence of $\lim _{n \rightarrow \infty} \operatorname{grad} g\left(u_{n}\right)$ in $H$. This implies

$$
\begin{aligned}
0 & \geq \varlimsup_{n \rightarrow \infty}\left(\left\langle L\left(u_{n}-u\right), u_{n}-u\right\rangle+\left\langle\operatorname{grad} g\left(u_{n}\right)-\operatorname{grad} g(u), u_{n}-u\right\rangle\right) \\
& =\varlimsup_{n \rightarrow \infty}\left\langle L\left(u_{n}-u\right), u_{n}-u\right\rangle .
\end{aligned}
$$

Now we take the decomposition $u_{n}=u_{n 1}+u_{n 2}, u=u_{1}+u_{2}, u_{i}, u_{n i} \in H_{i}$, $i=1,2$, where $H_{i}$ are closed subspaces of $H, H=H_{1} \oplus H_{2}$, which are invariant for $L$ and $\sigma\left(L \mid H_{1}\right) \subset(\gamma, \infty), \sigma\left(L \mid H_{2}\right) \subset(-\infty, 0]$ for $\gamma>0$. We know $\operatorname{dim} H_{2}<\infty$. Hence $u_{n 2} \rightarrow u_{2}$. This gives

$$
0 \geq \varlimsup_{n \rightarrow \infty}\left\langle L\left(u_{n 1}-u_{1}\right), u_{n 1}-u_{1}\right\rangle \geq \gamma \varlimsup_{n \rightarrow \infty}\left|u_{n 1}-u_{1}\right|^{2} .
$$

Hence $u_{n} \rightarrow u$.

Now we hint at a possible extension of the theory of Morse-Conley index in [1]. Let us assume that $f \in C^{1}(H, \mathbb{R})$ satisfies all assumptions of Theorem 2.1. Then we consider $f_{\varepsilon} \in C^{1}(H, \mathbb{R})$ introduced in the above proofs for any $\varepsilon>0$ sufficiently small. We already know that this function satisfies the P.S. condition. Hence there is a variational system $\left\{\Gamma_{\varepsilon}, \eta_{\varepsilon}\right\}$ relative to $f_{\varepsilon}$ in the sense of [1]. So the generalized Morse-Conley index can be defined for any $f_{\varepsilon}$, i.e. there is a family $\Sigma_{\varepsilon}$ of subsets of $H$ and a map $i_{\varepsilon}: \Sigma_{\varepsilon} \rightarrow \mathcal{S}$ for any $\varepsilon>0$ sufficiently small. Here $\mathcal{S}$ is the set of formal power series in $t$ with nonnegative coefficients. Moreover, by analysing the proof of $[1$, Lemma 3.1] there is $R>0$ such that $B_{R}=\{x \in H|| u \mid<R\} \in \Sigma_{\varepsilon}$ and $i_{\varepsilon}\left(B_{R}\right)=t^{\text {index } L}$. More generally, let $S \subset H$ be a bounded subset such that $S \in \Sigma_{\varepsilon}$ for any $\varepsilon>0$ small. By [1, Theorem 1.5], the index $i_{\varepsilon}(S)$ is independent of $\varepsilon$. We can introduce the following

Definition 2.9. Suppose $f$ satisfies the assumptions of Theorem 2.1. 
Let $\Sigma$ be the set of all bounded subsets $S \subset H$ such that $S \in \Sigma_{\varepsilon}$ for any $\varepsilon>0$ small. Then the generalized Morse-Conley index of $S \in \Sigma$ relative to $f$ is defined by $i(S)=i_{\varepsilon}(S)$.

Theorem 2.10. If $i(S) \neq 0$ then $0 \in \operatorname{grad} f(\operatorname{con} S)$. Here con $S$ is the closed convex hull of $S$.

Proof. Since $i(S) \neq 0$ we have $i_{\varepsilon}(S) \neq 0$. So there is $u_{\varepsilon} \in S$ such that $\operatorname{grad} f_{\varepsilon}\left(u_{\varepsilon}\right)=0$. Since any closed convex subset of $H$ is weakly closed, there is a solution of $\operatorname{grad} f=0$ in $\operatorname{con} S$ by the same arguments as in the proof of Theorem 2.1 .

By using Theorem 2.10 with $S=B_{R}$ for $R>0$ sufficiently large, we have another proof of Theorem 2.1.

We note that the invertibility of $L$ is not used in the definition of $i(S)$. Only the property $\operatorname{dist}(0, \sigma(L) \backslash\{0\})>0$ is essential. Then $L+\varepsilon \mathbb{I}$ is invertible for any $\varepsilon>0$ small.

TheOREM 2.11. Definition 2.9 is meaningful provided that $f \in C^{1}(H, \mathbb{R})$ is such that $f(u)=\frac{1}{2}\langle L u, u\rangle+g(u)$ for a bounded linear self-adjoint operator $L$ satisfying $\operatorname{dist}(0, \sigma(L) \backslash\{0\})>0$, and the assumptions (ii)-(iii) of Theorem 2.1 are satisfied as well.

Of course, Theorem 2.10 is also true for the case of Theorem 2.11.

Finally, we give a result on the existence of additional critical points for functions with a finite number of critical points, motivated by [4].

THEOREM 2.12. Let $f \in C^{1}(H, \mathbb{R})$ satisfy the P.S. condition and assume $f(u)=\frac{1}{2}\langle L u, u\rangle+g(u)$, where $L$ is an invertible self-adjoint bounded linear operator for which the index exists and $\operatorname{grad} g(x)=o(|x|)$ as $|x| \rightarrow \infty$. Suppose that $f$ has only a finite number of critical points $x_{1}, \ldots, x_{k}$ and all of them are nondegenerate. Then at least one of them satisfies

$$
\text { index } x_{i}=\operatorname{index} L \text {. }
$$

Proof. The proof follows immediately from [1, Corollary 2.10, Theorem 2.8 and Lemma 3.1].

Corollary 2.13. Let $f \in C^{1}(H, \mathbb{R})$ satisfy the P.S. condition and assume $f(u)=\frac{1}{2}\langle L u, u\rangle+g(u)$, where $L$ is an invertible self-adjoint bounded linear operator for which the index exists and $\operatorname{grad} g(x)=o(|x|)$ as $|x| \rightarrow \infty$. Suppose that $f$ has nondegenerate critical points $x_{1}, \ldots, x_{k}$ satisfying

$$
\text { index } x_{i} \neq \operatorname{index} L \quad \forall i=1, \ldots, k \text {. }
$$

Then $f$ has another critical point.

Corollary 2.13 can be extended in the sense of Theorem 2.6 as follows. 
TheOREM 2.14. Let $L: H \rightarrow H$ be a Fredholm, self-adjoint bounded linear operator for which the index exists and $\operatorname{dim} \operatorname{ker} L>0$. Let $H_{1} \oplus \operatorname{ker} L=$ $H$ be the orthogonal decomposition with the orthogonal projection $P: H \rightarrow$ $\operatorname{ker} L$. Suppose that $f \in C^{1}(H, \mathbb{R})$ satisfies the following assumptions:

(i) $f(u)=\frac{1}{2}\langle L u, u\rangle+g(u)$;

(ii) there is a constant $M>0$ such that $|\operatorname{grad} g(x)| \leq M$ for all $x \in H$;

(iii) $\operatorname{grad} f$ has the property $S_{+}$;

(iv) there is a continuous map $\omega: S_{1}=\{v \in \operatorname{ker} L|| v \mid=1\} \rightarrow H$ such that

$$
\lim _{r \rightarrow+\infty} \operatorname{grad} g(u+r v)=\omega(v)
$$

uniformly in both $v \in S_{1}$ and $u \in \mathcal{A}$ for any fixed bounded subset $\mathcal{A}$ of $H_{1}$;

(v) there is no solution of the equation $\lambda v+P \omega(v)=0$ with $v \in S_{1}$ and $\lambda \geq 0$;

(vi) $f$ has nondegenerate critical points $x_{1}, \ldots, x_{k}$ such that

$$
\operatorname{index} x_{i} \neq \operatorname{index} L \quad \forall i=1, \ldots, k .
$$

Then $f$ has another critical point.

Proof. We apply Corollary 2.13 to the functions $f_{\varepsilon}=f+\varepsilon \psi$ with $\varepsilon>0$ sufficiently small, where $\psi \in C^{1}(H, \mathbb{R})$ is a convex function equal to 0 in an open neighbourhood $\mathcal{U} \subset H$ of $\left\{x_{1}, \ldots, x_{k}\right\}$ and $\psi(z)=|z|^{2} / 2$ for any $z$ sufficiently large. Moreover, we can assume that $\operatorname{grad} \psi(B)$ is bounded for any bounded subset $B \subset H$. Such a function $\psi$ can be defined by the formula $\psi(z)=\tau\left(|z|^{2} / 2\right)$ for a function $\tau \in C^{\infty}(\mathbb{R}, \mathbb{R})$ such that $\tau^{\prime} \geq 0, \tau^{\prime \prime} \geq 0$ and

$$
\tau(x)= \begin{cases}0 & \text { for }|x| \leq \max _{1 \leq i \leq k}\left|x_{i}\right|^{2} / 2+1, \\ x & \text { for }|x| \geq \max _{1 \leq i \leq k}\left|x_{i}\right|^{2} / 2+3 .\end{cases}
$$

Of course, such a function $\tau$ exists.

Since $\operatorname{grad} f$ has the property $S_{+}$and $\operatorname{grad} \psi$ is a monotone operator, we see that grad $f_{\varepsilon}$ has the property $S_{+}$as well. It is clear that $x_{1}, \ldots, x_{k}$ are the only critical points of $f_{\varepsilon}$ in some open set $\mathcal{U}_{1} \subset \mathcal{U}$, and they are nondegenerate with indices index $x_{i}, i=1, \ldots, k$. Moreover, grad $f_{\varepsilon}$ has the linear asymptote $L+\varepsilon \mathbb{I}$ at infinity. Now we apply Corollary 2.13 , by following simultaneously the proof of Theorem 2.5, to obtain a critical point of $f_{\varepsilon}$ in the set $\mathcal{B} \backslash \mathcal{U}_{1}$, where $\mathcal{B}$ is a sufficiently large, fixed ball. By letting $\varepsilon \rightarrow 0_{+}$ as in the proof of Theorem 2.6, we find a nontrivial critical point of $f$. The proof is finished.

Remark 2.15. If, in Theorem 2.14, we assume additionally that

(vii) $x_{1}=0$ and $f$ is $C^{2}$-smooth in an open neighbourhood of $\left\{x_{2}, \ldots, x_{k}\right\}$, then we can consider in the proof the usual, more convenient approximation $f_{\varepsilon}(z)=f(z)+\varepsilon|z|^{2} / 2$. Indeed, we can now use the implicit function theorem 
to show the existence of an open set $\mathcal{U}_{1}$ containing $\left\{x_{1}, \ldots, x_{k}\right\}$ such that $\operatorname{grad} f_{\varepsilon}$ has only $k$ critical points in $\mathcal{U}_{1}$, which are nondegenerate with indices index $x_{i}, i=1, \ldots, k$.

So, similarly to Theorem 2.3 , if grad $f$ is only pseudomonotone in Theorems 2.6 and 2.14 instead of having the property $S_{+}$, but (vii) additionally holds in Theorem 2.14 then $f$ almost has another critical point different from the given nondegenerate ones, i.e. there are open, bounded neighbourhoods $U_{1}, U_{2}$ of the set of all given nondegenerate critical points of $f$ such that $U_{1} \subset U_{2}$ and $\operatorname{grad} f=0$ is almost solvable on $U_{2} \backslash U_{1}$ (see Definition 2.2).

Remark 2.16. If grad $f$ is only quasimonotone (pseudomonotony implies quasimonotony; see $[3$, p. 946]) in all the above theorems, and (vii) of Remark 2.15 holds, in addition, in Theorem 2.14, then critical points of $f$ sought in these theorems almost exist in the sense of Definition 2.2 and Remark 2.15. This follows from the observation that the sum of a quasimonotone operator with $\varepsilon \mathbb{I}, \varepsilon>0$, has the property $S_{+}$. So $\operatorname{grad} f_{\varepsilon}=\operatorname{grad} f+\varepsilon \mathbb{I}$ also has the property $S_{+}$, and we can repeat the above proofs as in the proof of Theorem 2.3. In particular, in Theorem 2.3 and Remark 2.15 pseudomonotony can be replaced by quasimonotony.

Summing up we see that quasimonotony (resp. pseudomonotony) is not enough for the existence of a critical point (resp. a nontrivial critical point). Pseudomonotony (resp. the property $S_{+}$) is sufficient to ensure that some almost critical points (resp. almost nontrivial critical points) converge to exact ones. This convergence is only weak in the case of pseudomonotony.

Re m a r $\mathrm{k}$ 2.17. The assumption (v) of Theorems 2.5 and 2.14 is satisfied provided that we suppose

$\left(\mathrm{v}^{\prime}\right)$ For any $v \in \operatorname{ker} L$ with $|v|=1$ there is a symmetric positive semidefinite matrix $M_{v}, M_{v} \in \mathcal{L}(\operatorname{ker} L)$, relative to the inner product on $H$, such that $\left\langle\omega(v), M_{v} v\right\rangle>0$.

If there is an orthogonal basis $\left\{e_{i}\right\}$ of $\operatorname{ker} L$ such that for all $v \in \operatorname{ker} L$ with $|v|=1$ there is an $i$ such that $\left\langle\omega(v), v_{i}\right\rangle>0$, where $v=\left(v_{j}\right)$ is the orthogonal decomposition (the coordinates) of $v$ with respect to $\left\{e_{i}\right\}$. Then, by taking $M_{v} w=w_{i}$ for $w \in \operatorname{ker} L$, the assumption $\left(\mathrm{v}^{\prime}\right)$ holds. Of course, it holds if $\langle\omega(v), v\rangle>0$ for $v \in \operatorname{ker} L$ with $|v|=1$.

3. Applications. In this section, we use the above abstract results to prove existence results for several differential equations.

The first example is

$$
\begin{aligned}
& \frac{\partial F}{\partial x}\left(t, u(t), u^{\prime}(t)\right)-\frac{\partial}{\partial t}\left(\frac{\partial F}{\partial y}\left(t, u(t), u^{\prime}(t)\right)\right)=0, \\
& u: \mathbb{R} \rightarrow \mathbb{R} \text { is } 2 \pi \text {-periodic, }
\end{aligned}
$$


where $F \in C^{1}(\mathbb{R} \times \mathbb{R} \times \mathbb{R}, \mathbb{R})$ and $F$ is $2 \pi$-periodic in $t$. The equation (3.1) is the Euler equation of the functional

$$
f(u)=\int_{0}^{2 \pi} F\left(t, u(t), u^{\prime}(t)\right) d t .
$$

Theorem 3.1. Assume that

(i) there are numbers $a, b, d$ with $d>0$ satisfying

$$
\begin{aligned}
& \left|\frac{\partial F}{\partial x}(t, x, y)-a x-b y\right| / \sqrt{x^{2}+y^{2}} \rightarrow 0 \quad \text { as }|x|+|y| \rightarrow \infty \\
& \left|\frac{\partial F}{\partial y}(t, x, y)-b x-d y\right| / \sqrt{x^{2}+y^{2}} \rightarrow 0 \quad \text { as }|x|+|y| \rightarrow \infty
\end{aligned}
$$

uniformly in $t$;

(ii) $F(t, x, y)=\Phi(t, x, y)+\phi(t, x)$, where $\Phi$ is convex in $(x, y)$ for any fixed $t$, and $\Phi, \phi$ are $C^{1}$-smooth and $2 \pi$-periodic in $t$.

If $-a / d \notin\left\{0,1^{2}, 2^{2}, \ldots\right\}$, then (3.1) has at least one $2 \pi$-periodic weak solution u, i.e.

$$
\langle T(u), v\rangle=\int_{0}^{2 \pi}\left(\frac{\partial F}{\partial x}\left(t, u, u^{\prime}\right) v+\frac{\partial F}{\partial y}\left(t, u, u^{\prime}\right) v^{\prime}\right) d t=0
$$

for each $v$ in

$$
H=\left\{u: \mathbb{R} \rightarrow \mathbb{R} \mid u \text { is } 2 \pi \text {-periodic, }|u|^{2}=\int_{0}^{2 \pi}\left(u^{2}+\left(u^{\prime}\right)^{2}\right) d t<\infty\right\} .
$$

Here $T(u) \in H^{*}=H$.

Pro of. We apply Theorem 2.1 by setting

$$
\begin{aligned}
\langle L u, u\rangle & =\int_{0}^{2 \pi}\left(a u^{2}+d\left(u^{\prime}\right)^{2}\right) d t, \\
g(u) & =\int_{0}^{2 \pi}\left(F\left(t, u, u^{\prime}\right)-\frac{a u^{2}+d\left(u^{\prime}\right)^{2}}{2}\right) d t .
\end{aligned}
$$

We see that $L v=0$ if $a v=d v^{\prime \prime}, v \in H$. Since $-a / d \notin\left\{0,1^{2}, 2^{2}, \ldots\right\}$ we obtain $v=0$. Hence the assumption (i) of Theorem 2.1 is satisfied.

Furthermore, the condition (i) of the present theorem implies for any $r>0$ the existence of a constant $C(r)$ such that 


$$
\begin{aligned}
\left|\frac{\partial F}{\partial x}(t, x, y)-a x-b y\right| & \leq r \sqrt{x^{2}+y^{2}}+C(r), \\
\left|\frac{\partial F}{\partial y}(t, x, y)-b x-d y\right| & \leq r \sqrt{x^{2}+y^{2}}+C(r),
\end{aligned}
$$

for all $t, x, y \in \mathbb{R}$. Note that

$$
D g(u) v=\int_{0}^{2 \pi}\left(\left(\frac{\partial F}{\partial x}\left(t, u, u^{\prime}\right)-a u-b u^{\prime}\right) v+\left(\frac{\partial F}{\partial y}\left(t, u, u^{\prime}\right)-b u-d u^{\prime}\right) v^{\prime}\right) d t .
$$

By the Cauchy-Schwarz inequality we obtain

$$
\begin{aligned}
|D g(u) v| & \leq \sqrt{2} \int_{0}^{2 \pi}\left(r \sqrt{u^{2}+\left(u^{\prime}\right)^{2}}+C(r)\right) \sqrt{v^{2}+\left(v^{\prime}\right)^{2}} d t \\
& \leq \sqrt{2}(r|u|+\sqrt{2 \pi} C(r))|v| .
\end{aligned}
$$

This gives

$$
|\operatorname{grad} g(u)| \leq \sqrt{2}(r|u|+\sqrt{2 \pi} C(r)),
$$

and the assumption (ii) of Theorem 2.1 holds.

The pseudomonotony of grad $f$ follows from the decomposition $F(t, x, y)$ $=\Phi(t, x, y)+\phi(t, x)$, where $\Phi$ is convex in $(x, y)$ for any fixed $t$, by using $[6$, Theorem 3.3.42]. Note that the conditions (3.1.3), (3.1.4) and (3.3.37) of [6, Theorem 3.3.42] are satisfied for our case with $p=2$. The proof is finished.

TheOREM 3.2. Suppose that the condition (i) of Theorem 3.1 holds and

(ii) $\frac{\partial F}{\partial y}(t, x, y)$ is nondecreasing in $y$ for each $t, x$.

(Note that the assumption (ii) of Theorem 3.1 clearly implies the present assumption (ii).) Moreover, assume $F$ is $C^{2}$-smooth at $(0,0)$ and

$$
\begin{gathered}
F(t, x, y)=p x^{2}+2 s x y+q y^{2}+o\left(|x|^{2}+|y|^{2}\right) \quad \text { near } x=y=0 . \\
\text { If } q>0,-p / q \notin\left\{0,1^{2}, 2^{2}, \ldots\right\},-a / d \notin\left\{0,1^{2}, 2^{2}, \ldots\right\} \text { and } \\
\#\left\{n \in\{0,1,2, \ldots\} \mid n^{2}<-p / q\right\} \neq \#\left\{n \in\{0,1,2, \ldots\} \mid n^{2}<-a / d\right\},
\end{gathered}
$$

then (3.1) almost has a nonzero solution (see Theorem 2.3). Here \# $\mathcal{A}$ means the number of elements of a finite set $\mathcal{A}$.

Proof. We apply Theorem 2.3 and Remark 2.16. We have

$$
\begin{aligned}
D^{2} f(0)\left(v_{1} ; v_{2}\right) & =2 \int_{0}^{2 \pi}\left(p v_{1} v_{2}+s\left(v_{1} v_{2}^{\prime}+v_{1} v_{2}^{\prime}\right)+q v_{1}^{\prime} v_{2}^{\prime}\right) d t \\
& =2 q \int_{0}^{2 \pi}\left(\frac{p}{q} v_{1} v_{2}+v_{1}^{\prime} v_{2}^{\prime}\right) d t .
\end{aligned}
$$


Hence

index Hess $f(0)=\#\{n \in\{0\} \mid n<-p / q\}+2 \#\left\{n \in\{1,2, \ldots\} \mid n^{2}<-p / q\right\}$, index $L=\#\{n \in\{0\} \mid n<-a / d\}+2 \#\left\{n \in\{1,2, \ldots\} \mid n^{2}<-a / d\right\}$.

We see that the conditions (i)-(ii) of Theorem 2.3 are satisfied. Finally, the quasimonotony of grad $f$ holds again by [6, Theorem 3.3.42]. The proof is finished.

Remark 3.3. If the assumption (ii) of Theorem 3.1 is weakened to the assumption (ii) of Theorem 3.2, then (3.1) almost has a solution (see Remark 2.16).

If the assumption (ii) of Theorem 3.2 is strengthened to

$$
\frac{\partial F}{\partial y}(t, x, y) \text { is increasing in } y \text { for each } t, x,
$$

then by [6, Theorem 3.3.42] the operator $\operatorname{grad} f$ of Theorem 3.2 has the property $S_{+}$. Then we know that $f$ also satisfies the P.S. condition, and so (3.1) has a nonzero solution. This result is well known (see $[1,4]$ ).

Theorem 3.4. Assume that

(i) there are positive constants $M, d$ satisfying

$$
\left|\frac{\partial F}{\partial x}(t, x, y)\right|<M, \quad\left|\frac{\partial F}{\partial y}(t, x, y)-d y\right|<M \quad \forall(t, x, y) ;
$$

(ii) $\frac{\partial F}{\partial y}(t, x, y)$ is increasing in $y$ for each $t, x$;

(iii) $F$ is $C^{2}$-smooth at $(0,0)$ and

$$
F(t, x, y)=p x^{2}+2 s x y+q y^{2}+o\left(|x|^{2}+|y|^{2}\right) \quad \text { near } x=y=0 ;
$$

(iv) there are continuous functions $\gamma: \mathbb{R} \rightarrow \mathbb{R}, f_{ \pm}: \mathbb{R} \rightarrow \mathbb{R}$ and $\alpha$ : $\mathbb{R} \times \mathbb{R} \rightarrow \mathbb{R}$, where $f_{ \pm}, \alpha$ are $2 \pi$-periodic in $t$, such that

$$
\begin{gathered}
\lim _{x \rightarrow \pm \infty} \alpha(t, x)=f_{ \pm}(t) \quad \text { uniformly in } t, \quad \lim _{x \rightarrow+\infty} \gamma(x)=0, \\
\left|\frac{\partial F}{\partial x}(t, x, y)-\alpha(t, x)\right| \leq \gamma(|x|)(|y|+1) \quad \forall(t, x, y), \\
\left|\frac{\partial F}{\partial y}(t, x, y)-d y\right| \leq \gamma(|x|)(|y|+1) \quad \forall(t, x, y) .
\end{gathered}
$$

If $q>0, p<0,-p / q \notin\left\{0,1^{2}, 2^{2}, \ldots\right\}$ and

$$
\int_{0}^{2 \pi} f_{-}(t) d t<0, \quad \int_{0}^{2 \pi} f_{+}(t) d t>0
$$

then (3.1) has a nonzero solution. 
Proof. We apply Theorem 2.6 in the framework of Theorems 3.1 and 3.2. It is clear that

$$
\langle L u, v\rangle=\int_{0}^{2 \pi} d u^{\prime} v^{\prime} d t \quad \forall u, v \in H
$$

$\operatorname{ker} L=\{$ constant functions $\}, \quad\{v \in \operatorname{ker} L|| v \mid=1\}=\{ \pm 1 / \sqrt{2 \pi}\}$,

$$
H_{1}=\left\{v \in H \mid \int_{0}^{2 \pi} v(t) d t=0\right\} \text {. }
$$

Note that

$$
D g(u) v=\int_{0}^{2 \pi}\left(\frac{\partial F}{\partial x}\left(t, u, u^{\prime}\right) v+\left(\frac{\partial F}{\partial y}\left(t, u, u^{\prime}\right)-d u^{\prime}\right) v^{\prime}\right) d t
$$

By using the assumption (iv), we see that $\omega( \pm 1 / \sqrt{2 \pi})=\mathcal{K} f_{ \pm}$for this case, where $\mathcal{K} v$ is defined by the identity

$$
\int_{0}^{2 \pi}(\mathcal{K} v) u d t+\int_{0}^{2 \pi}(\mathcal{K} v)^{\prime} u^{\prime} d t=\int_{0}^{2 \pi} v u d t \quad \forall u \in H .
$$

So we have

$$
\begin{aligned}
\langle\omega( \pm 1 / \sqrt{2 \pi}), \pm 1 / \sqrt{2 \pi}\rangle & =\int_{0}^{2 \pi} \omega( \pm 1 / \sqrt{2 \pi})( \pm 1 / \sqrt{2 \pi}) d t \\
& = \pm \frac{1}{\sqrt{2 \pi}} \int_{0}^{2 \pi} \mathcal{K} f_{ \pm}(t) d t= \pm \frac{1}{\sqrt{2 \pi}} \int_{0}^{2 \pi} f_{ \pm}(t) d t .
\end{aligned}
$$

The assumption (v) of Theorem 2.5 is satisfied (see Remark 2.17). Furthermore, we have

$$
\begin{aligned}
D^{2} f(0)\left(v_{1} ; v_{2}\right) & =2 \int_{0}^{2 \pi}\left(p v_{1} v_{2}+s\left(v_{1} v_{2}^{\prime}+v_{1} v_{2}^{\prime}\right)+q v_{1}^{\prime} v_{2}^{\prime}\right) d t \\
& =2 q \int_{0}^{2 \pi}\left(\frac{p}{q} v_{1} v_{2}+v_{1}^{\prime} v_{2}^{\prime}\right) d t .
\end{aligned}
$$

Hence 0 is a nondegenerate critical point of $f$ and

$$
\text { index Hess } \begin{aligned}
f(0) & =\#\{n \in\{0\} \mid n<-p / q\}+2 \#\left\{n \in\{1,2, \ldots\} \mid n^{2}<-p / q\right\} \\
& >0=\text { index } L .
\end{aligned}
$$

By Remark 3.3, the operator grad $f$ has the property $S_{+}$. Summarizing we see that all assumptions of Theorem 2.6 hold. The proof is finished. 
THEOREM 3.5. If the assumptions (i), (iv) of Theorem 3.4 and (ii) of Theorem 3.1 hold and

$$
\int_{0}^{2 \pi} f_{-}(t) d t<0, \quad \int_{0}^{2 \pi} f_{+}(t) d t>0
$$

then (3.1) has a solution.

Pr o of. Apply Theorem 2.5 in the framework of Theorem 3.4.

Remark 3.6. If $\frac{\partial \Phi}{\partial y}(\cdot, \cdot, 0)=0, \Phi(\cdot, \cdot, 0)=0, \phi(t, x)=\phi(x)$ in the assumption (ii) of Theorem 3.1 and there are only a finite number of roots of $\phi^{\prime}(x)=0$, then each of these roots is a solution of (3.1). By applying Theorem 2.14, we can find a nonconstant solution of (3.1) under additional assumptions.

The higher-dimensional cases can be solved similarly. For instance, let us consider the equation

$$
\begin{aligned}
& -\sum_{i=1}^{n} \frac{\partial}{\partial x_{i}}\left(f_{i}(x, \nabla u)\right)+g(x, u)=0, \quad x \in \Omega \subset \mathbb{R}^{n}, \\
& u(x)=0, \quad x \in \partial \Omega, u: \Omega \rightarrow \mathbb{R},
\end{aligned}
$$

where $f_{i}(x, y)=\frac{\partial \bar{F}}{\partial y_{i}}(x, y), \bar{F} \in C^{2}\left(\mathbb{R}^{n} \times \mathbb{R}^{n}, \mathbb{R}\right), g \in C^{0}\left(\mathbb{R}^{n} \times \mathbb{R}, \mathbb{R}\right)$ and $\Omega$ is an open bounded subset of $\mathbb{R}^{n}$ with a smooth boundary $\partial \Omega$. Note the inner product on $\mathbb{R}^{n}$ is denoted by $(\cdot, \cdot)_{n}$.

\section{TheOREM 3.7. Assume that}

(i) $\left(\operatorname{Hess}_{y} \bar{F}(\cdot, \cdot) v, v\right)_{n} \geq 0$ for all $v \in \mathbb{R}^{n}$, where $\operatorname{Hess}_{y} \bar{F}=\left[\partial^{2} \bar{F} / \partial y_{i} \partial y_{j}\right]$ is a symmetric matrix;

(ii) there is a constant $c \in \mathbb{R}$ and a symmetric positive definite matrix $B: \mathbb{R}^{n} \rightarrow \mathbb{R}^{n}$ such that

$$
\operatorname{grad}_{y} \bar{F}(x, y)=B y+O(1), \quad g(x, z)=c z+O(1)
$$

as $|y|,|z| \rightarrow \infty$ uniformly in $x$;

(iii) $c \notin \sigma\left(\Delta_{B}\right.$ with the Dirichlet condition $)$, where we have set $\Delta_{B} u=$ $\sum_{i=1}^{n} \sum_{j=1}^{n} b_{i j} \partial^{2} u / \partial x_{i} \partial x_{j}$ and $B=\left[b_{i j}\right]$.

Then (3.2) has at least one weak solution $u \in H_{0}^{1}(\Omega)$, i.e.

$$
\int_{\Omega}\left(\sum_{i=1}^{n} f_{i}(x, \nabla u) \frac{\partial w}{\partial x_{i}}+g(x, u) w\right) d x=0, \quad \forall w \in H_{0}^{1}(\Omega) .
$$


P r o of. We apply Theorem 2.1 as in the proof of Theorem 3.1 by putting

$$
\begin{gathered}
\langle u, v\rangle=\int_{\Omega}(\nabla u, \nabla v)_{n} d x, \quad\langle L u, v\rangle=\int_{\Omega}(B \nabla u, \nabla v)_{n} d x+c \int_{\Omega} u v d x, \\
f(u)=\int_{\Omega} \bar{F}(x, \nabla u) d x+\int_{\Omega} G(x, u) d x, \quad G(x, z)=\int_{0}^{z} g(x, s) d s .
\end{gathered}
$$

Since the verification of the assumptions of Theorem 2.1 for this case is the same as for Theorem 3.1, the proof is finished.

Acknowledgments. I thank the referee for helpful comments and suggestions.

\section{References}

[1] V. Benci, Some applications of the generalized Morse-Conley index, Confer. Semin. Mat. Univ. Bari 218 (1987).

[2] J. Berkovits and V. Mustonen, On topological degree for mappings of monotone type, Nonlinear Anal. 10 (1986), 1373-1383.

[3] - - - An extension of Leray-Schauder degree and applications to nonlinear wave equations, Differential Integral Equations 3 (1990), 945-963.

[4] S. Li and J. Q. Liu, Morse theory and asymptotic linear Hamiltonian system, J. Differential Equations 78 (1989), 53-73.

[5] J. Mawhin and M. Willem, Critical Point Theory and Hamiltonian Systems, Springer, New York, 1989.

[6] J. Nečas, Introduction to the Theory of Nonlinear Elliptic Equations, Teubner, Leipzig, 1983.

[7] B. Przeradzki, An abstract version of the resonance theorem, Ann. Polon. Math. 53 (1991), 35-43.

MATHEMATICAL INSTITUTE

SLOVAK ACADEMY OF SCIENCES

ŠTEFÁNIKOVA 49

81473 BRATISLAVA, SLOVAKIA 